

\title{
MEDIUM EFFECTS ON KNOCKOUT OF DEEPLY BOUND NUCLEONS
}

\author{
Christian Miller, R. Abegg, D. Frekers, P. Green, K. Hicks, D. Hutcheon, \\ Mumtaz Ahmad, L. Greeniaus, P. Kitching, D. Mack, et al.
}

\section{- To cite this version:}

Christian Miller, R. Abegg, D. Frekers, P. Green, K. Hicks, et al.. MEDIUM EFFECTS ON KNOCKOUT OF DEEPLY BOUND NUCLEONS. Journal de Physique Colloques, 1990, 51 (C6), pp.C6-595C6-598. 10.1051/jphyscol:1990681 . jpa-00231096

\section{HAL Id: jpa-00231096 https://hal.science/jpa-00231096}

Submitted on 1 Jan 1990

HAL is a multi-disciplinary open access archive for the deposit and dissemination of scientific research documents, whether they are published or not. The documents may come from teaching and research institutions in France or abroad, or from public or private research centers.
L'archive ouverte pluridisciplinaire HAL, est destinée au dépôt et à la diffusion de documents scientifiques de niveau recherche, publiés ou non, émanant des établissements d'enseignement et de recherche français ou étrangers, des laboratoires publics ou privés. 


\author{
C.A. MILLER, R. ABEGG, D. FREKERS, P.W. GREEN, K. HICKS, D.A. HUTCHEON, \\ M. AHMAD* L.G. GREENIAUS* , P. KITCHING* , D. MACK ${ }^{*}$, W. J. MCDONALD* \\ W.C. OLSEN" ${ }^{*}$ Y. YE* N.S. CHANT** and R.'SCHUBANK* \\ TRIUMF, Vancouver B.C., V6T 2A3, Canada \\ "University of Alberta, Edmonton, Alberta, Canada \\ * University of Maryland, College Park, MD 20742, U.S.A. \\ *** University of Saskatchewan. Saskatoon, Saskatchewan, Canada
}

\title{
Résumé
}

Le pouvoir d'analyse de diffusion quasi-libre nucléon-nucléon est nettement différent de celui de l'interaction libre. Cette modification due au milieu nucléaire a été observé pour la premiere fois dans les expériences de diffusion inclusive. Nous montrons ici que des expériences en cinematique totalement exclusive permettent de mettre cet effet en évidence encore plus clairement. Cet avantage réside dans le fait de pouvoir sélectionner des nucléons liés dans des états bien définis à l'intérieur du noyau.

\begin{abstract}
The analyzing power for quasi-free nucleon-nucleon scattering has been found to differ substantially from that for the free interaction. This medium modification was first noticed in inclusive scattering experiments. In the present work, it has been demonstrated that kinematically complete exclusive measurements are capable of demonstrating this effect more clearly. This advantage arises by virtue of the possibility of selecting nucleons bound in states localized in the nuclear interior.
\end{abstract}

Modification of the nucleon-nucleon interaction in the nuclear medium has been found to be an important aspect of various reactions induced by intermediate energy protons. One of the most direct ways of studying such modifications is quasi-free scattering of nucleons by polarized protons. Inclusive measurements of spin observables in this reaction have yielded some results which depart significantly from expectations based on the free interaction, even after accounting for "obvious" effects such as internal motion of the nucleons and binding energy.

It is expected in the context of relativistic mean field theory and suggested by empirical fits of Dirac optical potentials to nuclear elastic scattering data that the potential experienced by a nucleon inside the nucleus includes large attractive Lorentz scaler and repulsive vector terms. It has been pointed out that the large negative scaler potential will reduce the effective mass of the nucleon upon which depend the Dirac spinors between which the nucleon-nucleon matrix elements are evaluated. In particular, the lower components of the spinors are expected to be enhanced. In one model of this effect, some spin observables are predicted to be significantly modified[1]. Most remakably, the analyzing power is substantially reduced. Although such medium effects are diluted in inclusive reactions because of emphasis of the nuclear surface where the density is not large, some indications of this medium effect have been observed[2].

In the investigation of medium effects on the basic interaction, we may expect that exclusive measurements can enjoy some significant advantages. First, the p-p and n-p interactions can be studied separately instead of being averaged together. Also, the half-off-shell kinematic conditions of each scattering event are determined experimentally instead of being averaged over, resulting in more explicit information. Most importantly, because the residual nucleus energy is determined experimentally, knockout from individual nuclear orbitals can be distinguished. To maximize medium effects, we may therefore select events corresponding to knockout from an orbital whose wave function has the bulk of its strength within the nuclear surface. The $1 \mathrm{~s}$ orbital satisfies this criterion as well as simplifies the interpretation of the data further by precluding any initial effective polarization of the struck nucleon due to distortion effects[3]. Kinematically emphasizing the nuclear interior avoids problems associated with surface-peaked reactions 
such as inclusive quasi-free scattering - for example the local density approximation is most suspect near the nuclear surface where the density gradient is large.

The capability of the exclusive reaction to emphasize the nuclear interior has been studied theoretically by incorporating into a non-relativistic DWIA calculation[4] the density dependence of Horowitz and Iqbal[1]. The radial dependence was explicitly included by relaxing the usual factorization approximation. It was found that modifications to certain spin observables were indeed predicted to be large - as much as a factor of two reduction in $A_{y}$, for example (see TRIUMF Experimental Proposal 473, C.A. Miller et al.(1987).

In an effort to exploit the advantages of the exclusive reaction, spin observables for knockout of $1 \mathrm{~s}$ nucleons have been measured for the first time. The choice of a $1 \mathrm{p}$ shell nucleus, ${ }^{16} \mathrm{O}$, as well as a beam energy of $500 \mathrm{Mev}$ were defined by the need to minimize the effects of absorption on the final state nucleons. In the first phase of the experiment, the analyzing power $A_{y}$ was determined while in a later run still being analyzed, the spin transfer parameters $D_{s^{\prime} s}, D_{s^{\prime} l}$ and $\mathrm{P}$ were measured.

The experiment was carried out with the polarized proton beam from the TRIUMF cyclotron. The Medium Resolution Spectrometer (MRS) was used to detect and identify a proton in coincidence with a lower energy proton which was detected in a crude magnetic spectrometer consisting of a dipole magnet with tracking from four vertical-drift chambers. The missing-energy resolution was adequate to easily resolve the $1 \mathrm{~s}$ knockout from the two $\mathrm{p}$ states and to resolve the $\mathrm{p}$ states from each other via peak fitting. Analyzing power measurements were done at MRS angles of $20^{\circ}, 25^{\circ}$ and $30^{\circ}$ while the spin transfer measurement was done only at $25^{\circ}$. In each case, the other detector arm was optimized for minimum recoil momentum at the $1 \mathrm{~s}$ separation energy of $40 \mathrm{MeV}$.

Figure 1 shows analyzing power results for knockout of 1 s protons from ${ }^{16} \mathrm{O}$. They are drastically reduced in comparison with the free values shown for the same two-body kinematic conditions. The degree of "quenching" of $A_{y}$ is larger than that found in inclusive measurements as well as expectations based on the density-dependent DWIA calculation mentioned above. Although the optical potentials for ${ }^{16} \mathrm{O}$ used here are only crude, they had little effect on the analyzing power in the absence of the density dependence. A more interesting comparison will be with the new finite-range Dirac-based DWIA calculation which implicitly includes modified Dirac spinors[5].

Analyzing power data for p-state knockout is shown in Figure 2. It reveals the familiar spin-orbit splitting due to the struck nucleon effective polarization induced by final state distortions. Hence it is manifested through the NN spin correlation parameter $A_{y y}$. The degree of splitting therefore is sensitive to medium modification of this NN observable. However, such interpretation will require careful comparison with a calculation using reliable optical potentials.

Preliminary indications from analysis of a portion of the spin transfer data are that medium modifications are present but not large. However there is a suggestion that the polarization parameter $\mathrm{P}$ departs significantly from the analyzing power $A_{y}$, an effect not predicted by the nonrelativistic calculations.

\section{References}

1. C.J. Horowitz and M.J. Iqbal, Phys. Rev. C33 (1986) 2059.

2. T.A. Carey et al., Phys. Rev. Lett. 53 (1984) 144.

C. Chan et al., Nucl. Phys. A510 (1990) 713.

3. G. Jacob et al., Nucl. Phys. A257 (1976) 517.

4. N.S. Chant et al., Phys. Rev. Lett. 43 (1979) 495.

N.S. Chant and P.G. Roos, Phys. Rev. C15 (1977) 57.

5. E.D. Cooper and O.V. Maxwell, Nucl. Phys. A493 (1989) 468. 


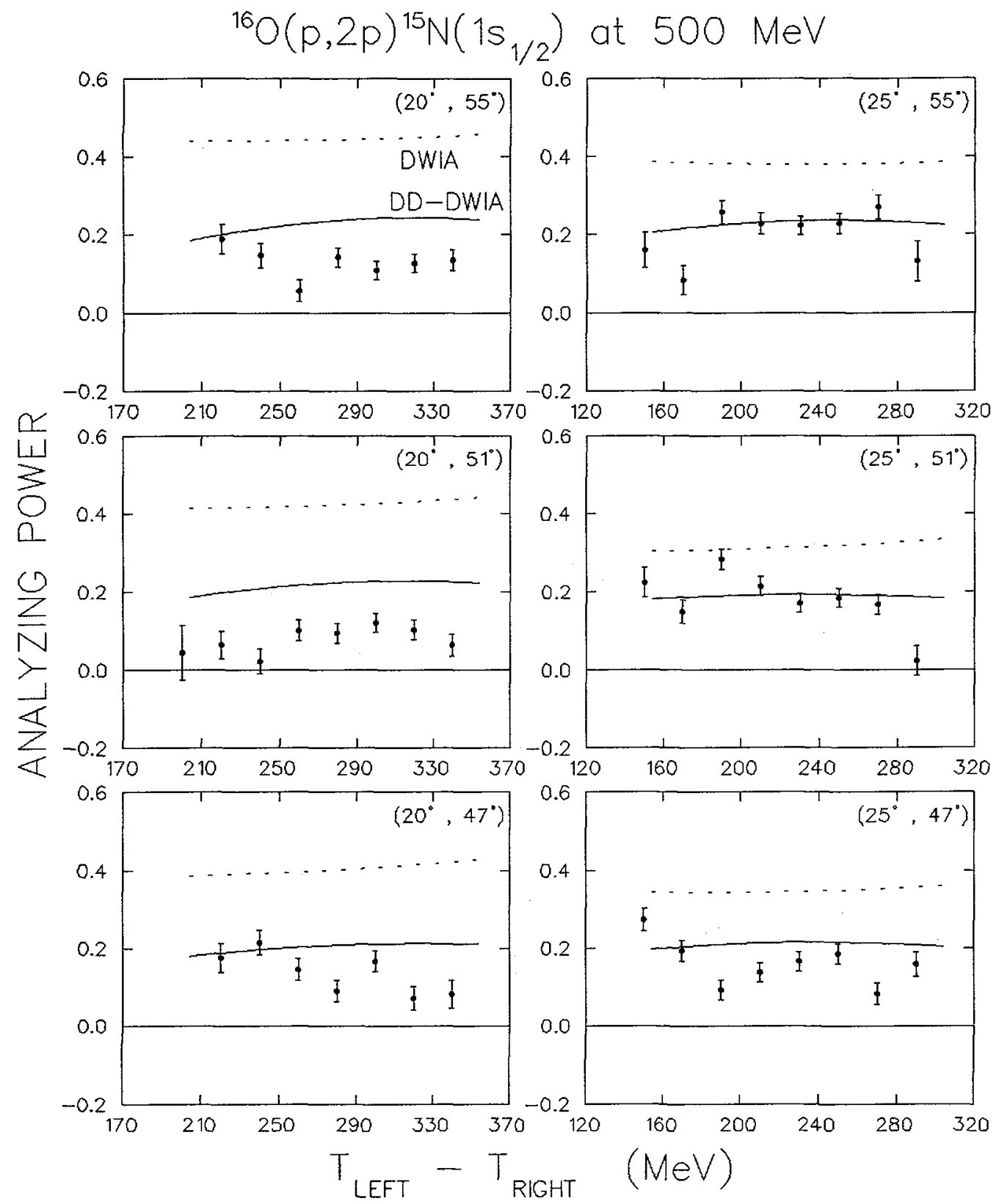

Fig. 1 - Analyzing powers for knockout of protons from the 1 s state in ${ }^{16} \mathrm{O}$, plotted versus the difference between the final state proton energies. The curves show Distorted Wave Impulse Approximation calculations. Relativistic density dependence is (not) included in the case of the (dashed) solid curves. The dashed curves differ little from the values for the free $p$-p interaction. 


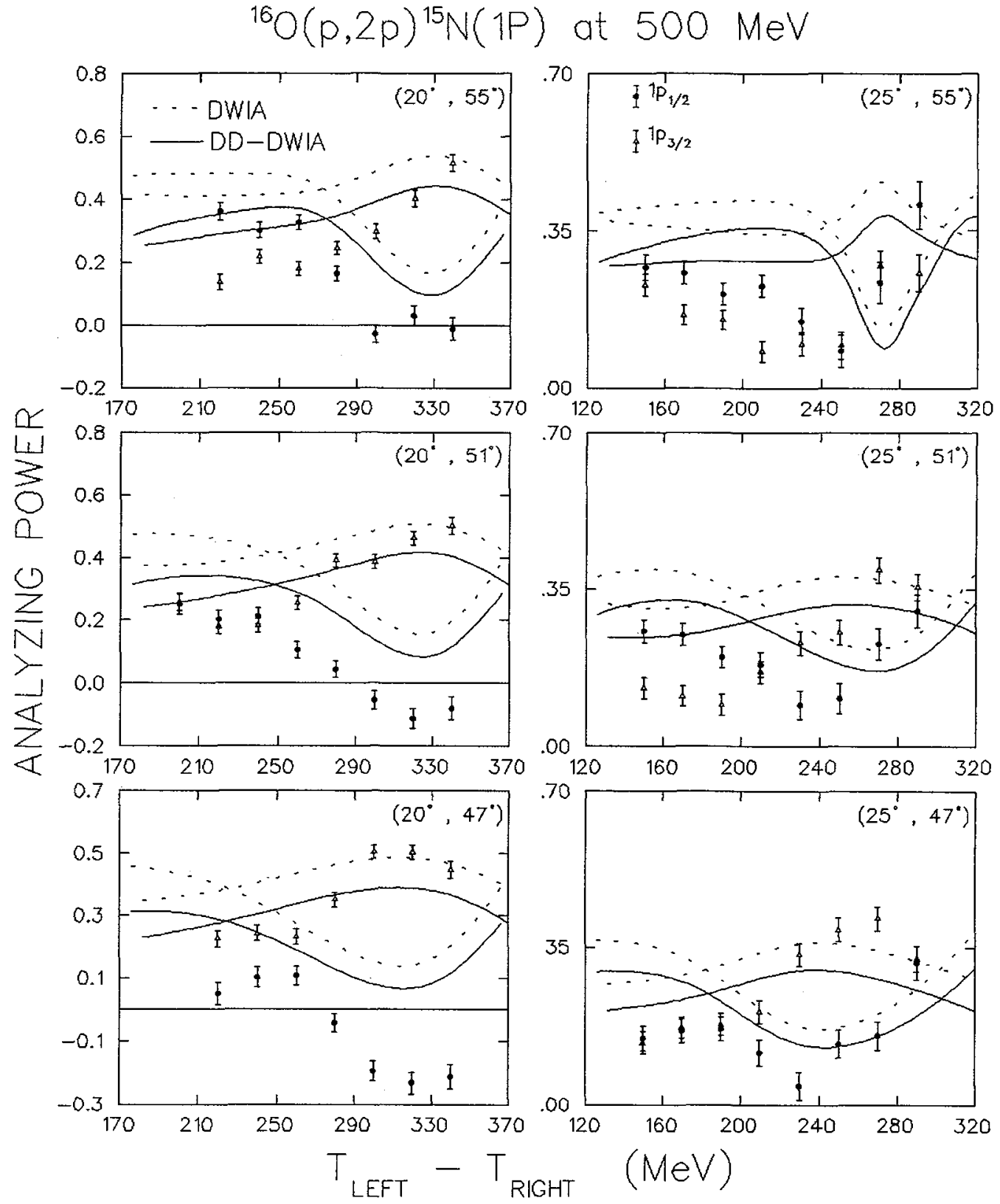

Fig. 2 - Analyzing powers for knockout of protons from the $1 \mathrm{p}$ states in ${ }^{16} \mathrm{O}$, plotted versus the difference between the final state proton energies. The curves have the same meaning as in fig. 1 . 